\title{
IMPLEMENTATION OF BOUNDARY CONDITIONS FOR OPTIMIZED HIGH-ORDER COMPACT SCHEMES
}

\author{
JAE WOOK KIM and DUCK JOO LEE \\ Korea Advanced Institute of Science and Technology. \\ Department of Aerospace Engineering, 373-1 Kusong-dong, Yusong-gu, Taejon, Korea
}

Received 6 May 1996

Revised 5 September 1996

\begin{abstract}
The optimized high-order compact (OHOC) finite difference schemes, proposed as central schemes are used for aeroacoustic computations on interior nodes. On near-boundary nodes, accurate noncentral or one-sided compact schemes are formulated and developed in this paper for general computations in domains with non-periodic boundaries. The near-boundary non-central compact schemes are optimized in the wavenumber domain by using Fourier error analysis. Analytic optimization methods are devised to minimize the dispersion and dissipation errors, and to obtain maximum resolution characteristics of the near-boundary compact schemes. With the accurate near-boundary schemes, the feasibility of implementing physical boundary conditions for the OHOC schemes are investigated to provide high-quality wave solutions. Characteristics-based boundary conditions and the free-field impedance conditions are used as the physical boundary conditions for direct computations of linear and nonlinear wave propagation and radiation. It is shown that the OHOC schemes present accurate wave solutions by using the optimized near-boundary compact schemes and the physical boundary conditions.
\end{abstract}

\section{Introduction}

The seven-point stencil high-order central compact finite difference schemes for evaluation of the first derivatives on interior nodes were optimized in the wavenumber domain by Kim and Lee. ${ }^{1}$ The analytic optimization methods were devised to minimize dispersion errors and obtain the maximum resolution characteristics of the central compact or non-compact schemes. ${ }^{1,2}$ With periodic boundaries and simple boundary conditions, the optimized highorder compact $(\mathrm{OHOC})$ schemes were used accurately to provide high-quality linear and nonlinear wave solutions. ${ }^{1,3-5}$

Many practical applications involve computations in domains with non-periodic boundaries. For these general computations by the OHOC (optimized high-order compact) schemes on interior nodes, accurate non-central or one-sided compact finite difference schemes should be incorporated on near-boundary nodes. Accurate evaluation of the first derivatives on the near-boundary nodes is important for the high-order and high-resolution schemes to provide high-quality numerical solutions. ${ }^{6}$ In general, when all the first derivatives of the Euler equations are evaluated by the high-order finite differences, the order of 
the resulting finite difference equations is higher than that of the original partial differential equations. Moreover, the high-order multidiagonal compact schemes may produce serious errors near the boundaries unless such accurate near-boundary compact schemes are implemented. The near-boundary schemes are, of necessity, non-central or one-sided differences and their error characteristics are both dispersive and dissipative, while those of the central compact schemes are only dispersive. Fourier analysis provides an effective way to quantify the dispersion and the dissipation errors of the near-boundary compact schemes. ${ }^{6}$ In this paper, the errors of the non-central near-boundary compact schemes are minimized in the wavenumber domain through the Fourier analysis and analytic optimization methods. In this way, the near-boundary compact schemes may be optimized to obtain maximum resolution characteristics and high accuracy.

When the first derivatives are evaluated accurately on the near-boundary nodes, correct and physical boundary conditions should be imposed in order to yield high-quality wave solutions. The high-order compact schemes are sensitive to boundary values in the evaluation of the first derivatives, so the quality of solutions, even on the interior nodes, are dependent on the accuracy of boundary conditions. For this reason, the correct physical boundary conditions as well as the accurate near-boundary schemes are essential to the actual applications of the OHOC schemes.

Recently, several suggestions for the physical boundary conditions have been proposed for unsteady aeroacoustic computations. These proposals can be classified in three categories, namely, (1) quasi one-dimensional characteristics, (2) decomposition of the solution into Fourier modes, and (3) asymptotic analysis of the governing equations for large distances. ${ }^{7}$ Thompson ${ }^{8,9}$ decomposed hyperbolic equations into wave modes of definite velocity and then specified boundary conditions for the incoming waves. The starting point of his analysis was the nonlinear Euler equations. The idea of his approach was that one-dimensional characteristic analysis could be performed by considering the transverse terms as a constant source term. The amplitudes of the outward propagating waves were defined entirely from the variables inside the computational domain, while those of the inward propagating waves were specified as the boundary conditions. These characteristics-based boundary conditions were utilized and developed by Poinsot and Lele ${ }^{10}$ for the Navier-Stokes (computations with non-dissipative algorithms. Giles ${ }^{11}$ derived boundary conditions based on the Fourier analysis of the linearized Euler equations. His basic idea was that the dispersion relation for the linear equations could be modified to prohibit propagation for waves with group velocities directed into the computational domain. Tam and Webb, ${ }^{2}$ Bayliss and Turkel, ${ }^{12}$ and Hagstrom and Hariharan ${ }^{13}$ considered boundary conditions based on an asimptotic analysis of the linearized Euler equations with constant mean flow in one direction. These approaches were effective when the position of acoustic source is specified exactly. The system of asymptotic equations could be reduced to the convective ware equations for pressure.

The present work is concerned with applications of the characteristics-based boundary conditions to the OHOC schemes. Thompson's inviscid wall and non-reflecting boundary conditions are used for the actual computations. Especially on radiation boundary, the 
free-field impedance conditions ${ }^{14}$ are imposed to correct the multi-dimensionality problem in Thompson's non-reflecting boundary conditions. The free-field impedance conditions can improve the non-reflecting characteristics of the radiation boundary conditions. In this paper, these physical boundary conditions are implemented for the OHOC schemes to provide high quality numerical solutions of linear and nonlinear wave propagation and radiation.

\section{Governing Equations}

The linear and nonlinear waves are computed from the Euler equations. The conservative form of axisymmetric and two-dimensional Euler equations in the Cartesian coordinates are considered in this paper.

$$
\frac{\partial \mathrm{Q}}{\partial t}+\frac{\partial \mathrm{E}}{\partial x}+\frac{\partial \mathrm{F}}{\partial y}+\alpha \mathrm{H}=0
$$

where

$$
\mathrm{Q}=\left[\begin{array}{c}
\rho \\
\rho u \\
\rho v \\
\rho e_{t}
\end{array}\right], \quad \mathrm{E}=\left[\begin{array}{c}
\rho u \\
\rho u^{2}+p \\
\rho u v \\
\left(\rho e_{t}+p\right) u
\end{array}\right], \quad \mathrm{F}=\left[\begin{array}{c}
\rho v \\
\rho u v \\
\rho v^{2}+p \\
\left(\rho e_{t}+p\right) v
\end{array}\right], \quad \mathrm{H}=\frac{1}{x}\left[\begin{array}{c}
\rho u \\
\rho u^{2} \\
\rho u v \\
\left(\rho e_{t}+p\right) u
\end{array}\right] .
$$

The axisymmetric and two-dimensional equations are formulated in cases where $\alpha=1$ and $\alpha=0$ respectively. The linear and nonlinear wave propagation and radiation are computed from Eq. (2.1) by using effective numerical algorithms and boundary conditions. The spatial derivative terms in Eq. (2.1) are evaluated by the OHOC schemes. For nonlinear wave computations, the artificial selective damping (ASD) terms ${ }^{4,5,15}$ can be added in the right side of Eq. (2.1) as a treatment of nonlinearity. The fourth-order low dissipation and dispersion Runge-Kutta (LDDRK) scheme is used as a time advancing method, which has two steps with 4-6 alternating stages. ${ }^{4,5,16}$ These numerical algorithms can be also applied to three-dimensional problems in generalized coordinates without any additional treatment.

\section{OHOC Schemes in a Domain with Non-Periodic Boundaries}

\subsection{OHOC schemes on interior nodes}

The main schemes presented here are generalizations of the Padé scheme of seven-point stencil as shown below ${ }^{1,2-5}$ :

$$
\beta f_{i-2}^{\prime}+\alpha f_{i-1}^{\prime}+f_{i}^{\prime}+\alpha f_{i+1}^{\prime}+\beta f_{i+2}^{\prime}=c \frac{f_{i+3}-f_{i-3}}{6 \Delta x}+b \frac{f_{i+2}-f_{i-2}}{4 \Delta x}+a \frac{f_{i+1}-f_{i-1}}{2 \Delta x} .
$$

This is the central difference formulation for the evaluation of the first derivatives on the interior nodes. The relations that determine the truncation order of this scheme are derived by using Taylor series expansion of Eq. (3.1). Only the eighth-order tridiagonal $(\beta=0)$ scheme and the tenth-order pentadiagonal $(\beta \neq 0)$ scheme have unique values of coefficients $a, b, c, \alpha$ and $\beta$, and these ones are of the highest order obtainable with Eq. (3.1). The other lower order schemes should have free coefficients that are not determined completely until 
more constraints are imposed, and these can be used to improve the resolution characteristics. Analytic and systematic constraints for the determination of the free coefficients are considered. The nature of these constraints is minimization of the dispersive (phase) errors in the wavenumber domain. Using these constraints, Kim and Lee ${ }^{1}$ succeeded in optimizing the compact schemes and showed that the optimized sixth-order tridiagonal (OSOT) and the fourth-order pentadiagonal (OFOP) schemes are more accurate than any other central compact schemes. In Ref. 1, the coefficients of the OSOT and OFOP scheme were presented as

- OSOT Scheme $(\beta=0)$ :

$$
\begin{aligned}
& a=1.568098211519709, \quad b=0.2716571074522698, \quad c=-0.02257678073547548 \\
& \alpha=0.4085892691182515 .
\end{aligned}
$$

- OFOP Scheme $(\beta \neq 0)$ :

$$
\begin{aligned}
& a=1.279672797796143, \quad b=1.051191982414920, \quad c=0.04475268855213291, \\
& \alpha=0.5900108167074074, \quad \beta=0.09779791767419070 .
\end{aligned}
$$

These optimum coefficients provide high-order accuracy and maximum resolution characteristics for the central compact schemes, and these schemes are proposed as the OHOC schemes. The maximum resolution characteristics of the OHOC schemes are compared with those of other standard central schemes in Fig. 1.

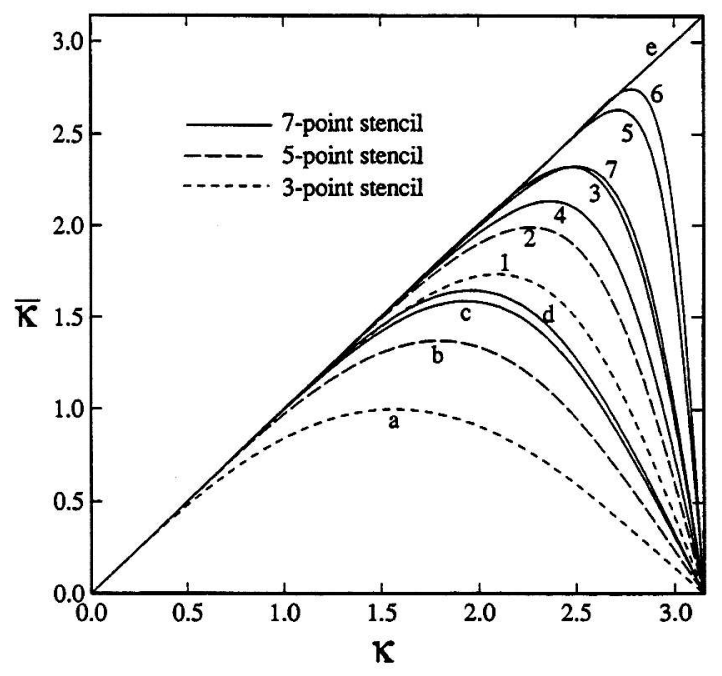

Fig. 1. Maximum resolution characteristics of $O H O C$ schemes in comparison with other standard central schemes: (a) second-order central differences, (b) fourth-order central differences, (c) sixth-order central differences, (d) DRP scheme in space, (e) exact differentiation, (1) standard Padé scheme, (2) sixth-order tridiagonal scheme (c $=0$ ), (3) OSOT scheme, (4) eight-order tridiagonal scheme, (5) Lele's fourth-order pentadiagonal scheme, (6) OFOP scheme,

(7) tenth-order pentadiagonal scheme. 


\subsection{Compact schemes on near-boundary nodes}

In this section, an analytic optimization of the non-central near-boundary compact schemes is considered for accurate computations in a domain with non-periodic boundaries. The OHOC schemes use seven-point stencil on the interior nodes. Thus three kinds of nearboundary schemes are required on three nodes $(i=0,1$ and 2$)$ from the boundary $(i=0)$. The near-boundary compact differences can be formulated as

$$
\begin{aligned}
& i=0: f_{0}^{\prime}+\alpha_{0,1} f_{1}^{\prime}+\beta_{0,2} f_{2}^{\prime}=\frac{1}{\Delta x} \sum_{j=0}^{3} a_{0, j} f_{j}, \\
& i=1: \alpha_{1,0} f_{0}^{\prime}+f_{1}^{\prime}+\alpha_{1,2} f_{2}^{\prime}+\beta_{1,3} f_{3}^{\prime}=\frac{1}{\Delta x} \sum_{j=0}^{4} a_{1, j} f_{j}, \\
& i=2: \beta_{2,0} f_{0}^{\prime}+\alpha_{2,1} f_{1}^{\prime}+f_{2}^{\prime}+\alpha_{2,3} f_{3}^{\prime}+\beta_{2,4} f_{4}^{\prime}=\frac{1}{\Delta x} \sum_{j=0}^{5} a_{2, j} f_{j},
\end{aligned}
$$

where, all the $\beta_{i, j}$ 's should be equal to zero for the tridiagonal schemes. These formulations are, of necessity, non-central differences and their error characteristics are both dispersive and dissipative. The two kinds of errors are analyzed simultaneously in the wavenumber domain by the Fourier analysis and the errors can be minimized by the analytic optimization methods, which are similar to those in Ref. 1 for the central compact schemes. The relations to determine the truncation orders of Eqs. (3.2)-(3.4) can be derived by using Taylor series expansion. In the following subsections, optimum values of the coefficients $\alpha_{i, j}$ 's, $\beta_{i, j}$ 's and $a_{i, j}$ 's are found to obtain maximum resolution characteristics and high accuracy of the near-boundary compact schemes.

\subsection{Fourier analysis of errors}

The dispersion and dissipation errors of Eqs. (3.2)-(3.4) are analyzed in the wavenumber $(\omega)$ domain. The Fourier analysis provides an effective way to quantify these errors and resolution characteristics of the differencing schemes, so that this quantification would be further used to guide an analytic optimization of the schemes. The Fourier transformations of the left and right sides of Eqs. (3.2)-(3.4) are expressed simply as

$$
\kappa[A(\kappa)+i B(\kappa)] \tilde{f}=[C(\kappa)+i D(\kappa)] \tilde{f},
$$

where, $\kappa$ (scaled wavenumber $)=\omega \Delta x$ and $i=\sqrt{-1}$. The functions $A, B, C$ and $D$ in Eq. (3.5) are specified for each schemes as

- $i=0$ :

$$
\begin{aligned}
& A(\kappa)=-\alpha_{0,1} \sin (\kappa)-\beta_{0,2} \sin (2 \kappa), \\
& B(\kappa)=1+\alpha_{0,1} \cos (\kappa)+\beta_{0,2} \cos (2 \kappa),
\end{aligned}
$$




$$
\begin{aligned}
& C(\kappa)=\sum_{j=0}^{3} a_{0, j} \cos (j \kappa), \\
& D(\kappa)=\sum_{j=0}^{3} a_{0, j} \sin (j \kappa) .
\end{aligned}
$$

- $i=1$ :

$$
\begin{aligned}
& A(\kappa)=\left(\alpha_{1,0}-\alpha_{1,2} \sin (\kappa)-\beta_{1,3} \sin (2 \kappa),\right. \\
& B(\kappa)=1+\left(\alpha_{1,0}+\alpha_{1,2}\right) \cos (\kappa)+\beta_{1,3} \cos (2 \kappa), \\
& C(\kappa)=\sum_{j=0}^{4} a_{1, j} \cos [(j-1) \kappa], \\
& D(\kappa)=\sum_{j=0}^{4} a_{1, j} \sin [(j-1) \kappa] .
\end{aligned}
$$

- $i=2$ :

$$
\begin{aligned}
& A(\kappa)=\left(\alpha_{2,1}-\alpha_{2,3} \sin (\kappa)+\left(\beta_{2,0}-\beta_{2,4}\right) \sin (2 \kappa),\right. \\
& B(\kappa)=1+\left(\alpha_{2,1}+\alpha_{2,3}\right) \cos (\kappa)+\left(\beta_{2,0}+\beta_{2,4}\right) \cos (2 \kappa), \\
& C(\kappa)=\sum_{j=0}^{5} a_{2, j} \cos [(j-2) \kappa], \\
& D(\kappa)=\sum_{j=0}^{5} a_{2, j} \sin [(j-2) \kappa] .
\end{aligned}
$$

From Eq. (3.5) with these functions, the scaled modified wavenumber $(\bar{\kappa})$ as a function of the scaled true wavenumber $(\kappa)$ for the near-boundary compact schemes are derived as

$$
\bar{\kappa}(\kappa)=\frac{A(\kappa) C(\kappa)+B(\kappa) D(\kappa)}{[A(\kappa)]^{2}+[B(\kappa)]^{2}}+i \frac{A(\kappa) D(\kappa)-B(\kappa) C(\kappa)}{[A(\kappa)]^{2}+[B(\kappa)]^{2}} .
$$

The modified wavenumbers for the non-central compact schemes can be divided into the real and imaginary part, while those for the central compact schemes consist of only a real term. The real and imaginary term in Eq. (3.6) express the dispersion and dissipation error characteristics respectively. The modified wavenumber defined in Eq. (3.6) is used for error analysis of the near-boundary compact schemes. To ensure that the Fourier transformation of the finite difference scheme is a good approximation of that of partial derivative, the modified wavenumber should coincide with the corresponding true wavenumber $(\bar{\kappa}=\kappa)$ in as wide a range of wavenumbers (i.e., $0 \leq \kappa<\pi$ ) as possible. As the modified wavenumber deviates from the true wavenumber, finite difference errors are produced. 


\subsection{Minimization of errors}

For the quantification of the dispersive and dissipative errors, an integrated error (weighted deviation) for the near-boundary compact schemes is defined as (see also Refs. 1, 2 and 17):

$$
\begin{aligned}
E & \equiv \int_{0}^{r \pi}|\bar{\kappa}-\kappa|^{2} W(\kappa) d \kappa \\
& =\int_{0}^{r \pi}\left\{[\operatorname{Re}(\bar{\kappa})-\kappa]^{2}+[\operatorname{Im}(\bar{\kappa})]^{2}\right\} W(\kappa) d \kappa,
\end{aligned}
$$

where $W(\kappa)$ is a weighting function, and $r$ is a factor to determine the optimization range $(0<r<1)$ under consideration. The weighting function in Eq. (3.7) would make the equation analytically integrable and the integrand to be weighted on the high wavenumber range (near $\pi$ ) where a lot of the dispersive and the dissipative errors exist. The integrated error $(E)$ defined in Eq. (3.7) is a function of the coefficients $\alpha_{i, j}$ 's, $\beta_{i, j}$ 's and $a_{i, j}$ 's. It is necessary to find the optimum values of the coefficients that would minimize the integrated error. The conditions that make ' $E$ ' a local minimum value are as follows:

$$
\begin{gathered}
\frac{\partial E}{\partial \alpha_{i, j}}=0 \\
\frac{\partial E}{\partial \beta_{i, j}}=0 \\
\frac{\partial E}{\partial a_{i, j}}=0 .
\end{gathered}
$$

Equations (3.8)-(3.10), which can minimize the integrated error, provide the constraints to obtain the optimum coefficients of the near-boundary compact schemes, in addition to the relations that determine the truncation orders.

\subsection{Weighting function}

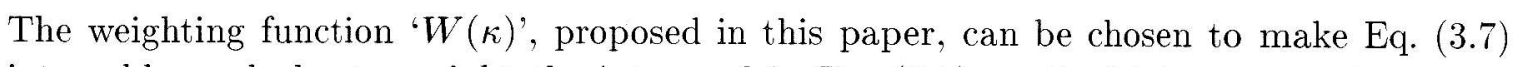
integrable, and also to weight the integrand in Eq. (3.7) on the high wavenumber range close to $\pi$ by multiplying an exponential term as

$$
W(\kappa)=\left\{\sqrt{[A(\kappa)]^{2}+[B(\kappa)]^{2}} e^{\kappa}\right\}^{2} .
$$

The result of weighting the integrated error to be in the high wavenumber range as opposed to the low wavenumber range is that the dispersive and the dissipative errors in the high wavenumber range can be more greatly reduced through the optimization procedure.

\subsection{Optimization ranges}

The optimization ranges for the near-boundary schemes should be adjusted to obtain maximum resolution characteristics of the schemes, i.e., by controlling the range factor $r$, the 
largest value of deviation $|\operatorname{Re}(\bar{\kappa})-\kappa|$ in the well-resolved range should be reduced to below $0.5 \%$ of the corresponding true wavenumber for tolerable accuracy.

As shown in Ref. 1, the effective optimization range factor for a scheme is dependent on the truncation order of the scheme. Once a suitable truncation order for a scheme is found, an optimization range factor for the scheme can then be determined. In Ref. 1, the truncation orders of the optimized central compact schemes were found by applying them to actual computations. The suitable truncation orders for the near-boundary compact schemes are found through the same procedure in the present work. With the truncation orders specified, the effective optimization range factors for the near-boundary compact schemes are determined as:

(i) Tridiagonal Schemes
(a) $i=0$ : second order : $r=0.401$,
(b) $i=1:$ fourth order : $r=0.622$,
(c) $i=2:$ sixth order : $r=0.881$.

(ii) Pentadiagonal Schemes
(a) $i=0$ : second order : $r=0.653$,
(b) $i=1$ : fourth order : $r=0.625$,
(c) $i=2$ : fourth order : $r=0.871$.

\subsection{Optimum coefficients}

The optimum coefficients $\alpha_{i, j}$ 's, $\beta_{i, j}$ 's and $a_{i, j}$ 's can be found for each scheme by using the analytic optimization methods, including the optimization range factors and the weighting function of Eq. (3.11). The constraints, Eq. (3.8)-(3.10), to minimize the integrated error and the relations to determine the truncation orders provide a system of linear algebraic equations by which the optimum coefficients are found. In this paper, the coefficients are presented for each near-boundary compact scheme as:

(i) Tridiagonal Schemes

(a) $i=0$ : second order :

$$
\begin{array}{ll}
a_{0,0}=-2.673444389108146, & a_{0,1}=1.468066764967325, \\
a_{0,2}=1.382688702485047, & a_{0,3}=-0.1773110783442254, \\
\alpha_{0,1}=2.701510934904742 . &
\end{array}
$$

(b) $i=1:$ fourth order :

$$
\begin{array}{ll}
a_{1,0}=-0.5088675754573845, & a_{1,1}=-0.7029878533366753 \\
a_{1,2}=1.040385365448375, & a_{1,3}=0.1867472036506759 \\
a_{1,4}=-0.01527714030499072, & \alpha_{1,0}=0.1532048781838751 \\
\alpha_{1,2}=0.7237110491082636 . &
\end{array}
$$

(c) $i=2:$ sixth order :

$$
a_{2,0}=-0.013127263621621, \quad a_{2,1}=-0.6038029221734134,
$$




$$
\begin{array}{ll}
a_{2,2}=-0.4395154246847092, & a_{2,3}=0.96090920472974, \\
a_{2,4}=0.1010303485585628, & a_{2,5}=-0.005493942808558833, \\
\alpha_{2,1}=0.2234544771621557, & \alpha_{2,3}=0.5530910456756884 .
\end{array}
$$

(ii) Pentadiagonal Schemes

(a) $i=0$ : second order :

$$
\begin{array}{ll}
a_{0,0}=-2.955167457862964, & a_{0,1}=-1.631750382194947, \\
a_{0,2}=4.280932270348171, & a_{0,3}=0.3059855697097409, \\
\alpha_{0,1}=4.573217321968533, & \beta_{0,2}=2.274853545662085 .
\end{array}
$$

(b) $i=1$ : fourth order :

$$
\begin{array}{ll}
a_{1,0}=-0.6437555190815847, & a_{1,1}=-0.2155624124985647, \\
a_{1,2}=1.39308006947385, & a_{1,3}=-0.4777810929596313, \\
a_{1,4}=-0.05598104493406903, & \alpha_{1,0}=0.2043562086111263, \\
\alpha_{1,2}=0.04640652276099101, & \beta_{1,3}=-0.337432463538152 .
\end{array}
$$

(c) $i=2:$ fourth order :

$$
\begin{array}{ll}
a_{2,0}=-0.1476189781906417, & a_{2,1}=-0.6598461743464279, \\
a_{2,2}=-0.1822518186408425, & a_{2,3}=0.6860603976309968, \\
a_{2,4}=0.29761855559004, & a_{2,5}=0.006038017956875419, \\
\beta_{2,0}=0.04025164856292263, & \alpha_{2,1}=0.4492362230014781, \\
\alpha_{2,3}=0.6599987763156845, & \beta_{2,4}=0.1050090455293296 .
\end{array}
$$
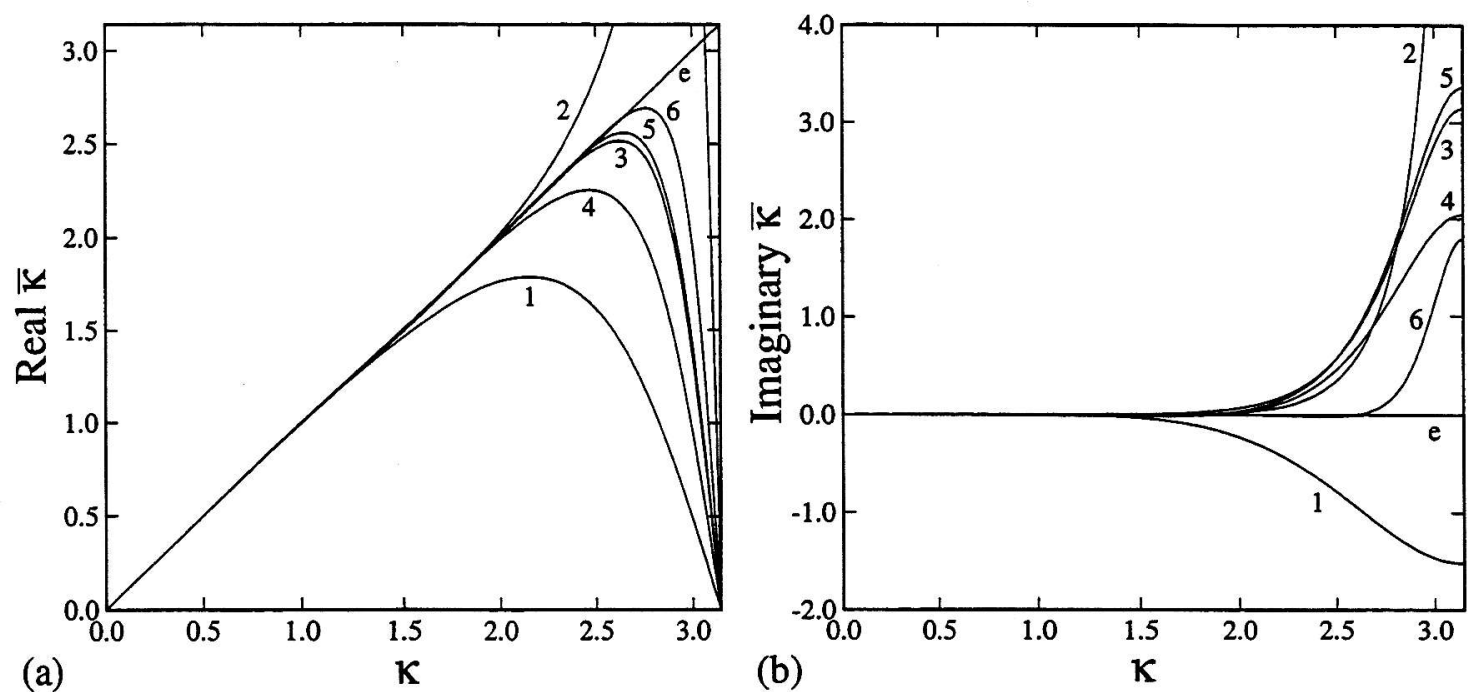

Fig. 2. Maximum resolution characteristics of the optimized near-boundary compact schemes: (a) dispersive characteristics, (b) dissipative characteristics: (1) second-order tridiagonal scheme on $i=0$, (2) fourth-order tridiagonal scheme on $i=1$, (3) sixth-order tridiagonal scheme on $i=2$, (4) second-order pentadiagonal scheme on $i=0$, (5) fourth-order pentadiagonal scheme on $i=1,(6)$ fourth-order pentadiagonal scheme on $i=2$, (e) exact differentiation. 
These optimum coefficients provide high accuracy and maximum resolution characteristics for the non-central compact schemes, and these schemes are proposed as the optimized near-boundary compact schemes. The optimized near-boundary compact schemes are combined and used with the OHOC schemes to evaluate the first derivatives accurately in the whole computational domain. The maximum resolution characteristics of the optimized near-boundary compact schemes are presented in Fig. 2.

The imaginary part of $\bar{\kappa}$ expresses the dissipation error characteristics, i.e., the stability of the optimized near-boundary compact schemes. In Fig. 2(b), the curves in the positive range are stable and those in the negative range are unstable. It is shown that the secondorder tridiagonal scheme on $i=0$ can be unstable, while the other optimized near-boundary compact schemes are all stable. In actual computations, however, it does not lead to remarkable unstableness by using the ASD terms.

\section{Physical Boundary Conditions}

\subsection{Boundary conditions for OHOC schemes}

When the first derivatives are evaluated accurately on near-boundary nodes by the optimized near-boundary compact schemes, correct physical boundary conditions should be imposed to yield high-quality wave solutions. The feasibility of implementing the physical boundary conditions for the OHOC schemes is investigated. In the present work, the characteristics-based boundary conditions and the free-field impedance conditions (see Ref. 14) are considered as the physical boundary conditions on an inviscid wall and nonreflecting radiation boundaries. Linear and nonlinear wave propagation and radiation is simulated by the OHOC schemes with the physical boundary conditions.

\subsection{Inviscid wall}

For the present work, Thompson's inviscid wall boundary conditions are used for computations in a domain with wall boundaries. Thompson decomposed the Euler equations into wave modes of definite velocity and then specified boundary conditions for the incoming waves. ${ }^{8,9}$ The amplitudes of the outward propagating waves are defined entirely from the variables inside the computational domain, while those of the inward propagating waves are specified as the boundary conditions. The procedures for implement these boundary conditions are described fully in Ref. 9.

\subsection{Non-reflecting radiation}

In a one-dimensional problem, the solution of the Euler equations can be constructed by means of the three sets of characteristics. The information concerning the solution is transmitted in space and time by these three characteristics. Thus, the characteristics provide a way to formulate the physical boundary conditions, and these can be the correct boundary conditions for the one-dimensional problem. However, one important drawback in the characteristics-based boundary conditions is that there are no true characteristics in two- or three-dimensional problems. As an approximation, one may ignore the multi-dimensionality 
of a problem at a boundary, and treat the problem as if it is locally one-dimensional with the direction normal to the boundary. The idea of Thompson's approach is that the onedimensional characteristic analysis can be performed by considering the transverse terms as a constant source term. For the radiation or outflow boundary conditions, this approximation has been found to lead to significant non-physical reflecting waves when the incident angles of the outward propagating waves are oblique to the boundary, or when there is a strong mean flow tangent to the boundary. ${ }^{18}$

For the present work, the characteristics-based radiation boundary conditions are improved for non-reflecting acoustic radiation. On the radiation boundary, the free-field impedance conditions are imposed to obtain the fluctuations of velocity and pressure, while the density is evaluated by Thompson's characteristics-based non-reflecting boundary conditions. The free-field impedance conditions can improve the non-reflecting characteristics of the radiation boundary conditions. The free-field impedance conditions are: (1) the acoustic wave radiating through the far boundary is nearly cylindrical or spherical, (2) the acoustic wave is thought to be locally planar with a non-dimensional acoustic impedance of 1 , and (3) the radiating process is locally isentropic. These are expressed by the following relations:

$$
\begin{aligned}
u_{\text {radial }}^{\prime} & =\frac{a_{o}}{\rho_{o}} \rho^{\prime}, \\
p^{\prime} & =a_{o}^{2} \rho^{\prime},
\end{aligned}
$$

where, $\rho^{\prime}, p^{\prime}$ and $u_{\text {radial }}^{\prime}$ are the fluctuations of density, pressure and radial velocity, respectively. ( $\rho_{o}$ and $a_{o}$ are the ambient density and the speed of sound respectively). These relations are used as the non-reflecting radiation boundary conditions for the present computations in accordance with the density fluctuation obtained by Thompson's non-reflecting boundary conditions.

\section{Applications to Linear and Nonlinear Waves}

\subsection{Linear waves}

The linear acoustic wave propagation and radiation due to a vibrating piston is simulated in axisymmetric coordinates. The conservative forms of axisymmetric Euler equations are used for the computations. The schematic diagram of the problem considered here is described in Fig. 3. It is a problem provided by the ICASE/LaRC Workshop on Benchmark Problems in Computational Aeroacoustics. ${ }^{16}$ The problem is suitable for verifying the effectiveness and accuracy of the optimized near-boundary compact schemes with the physical boundary conditions. The results of computations by the OSOT and the OFOP schemes are presented in Figs. 4 and 5 respectively. It is shown that the numerical solutions are in good agreement with the analytic solutions in the overall domain. The acoustic waves radiated well through the far-field boundaries with little reflecting waves, and propagated well along the inviscid wall keeping the wave fronts undistorted. However, it is expected that a long-time computation without imposing an analytic boundary condition (see Ref. 2) may produce 


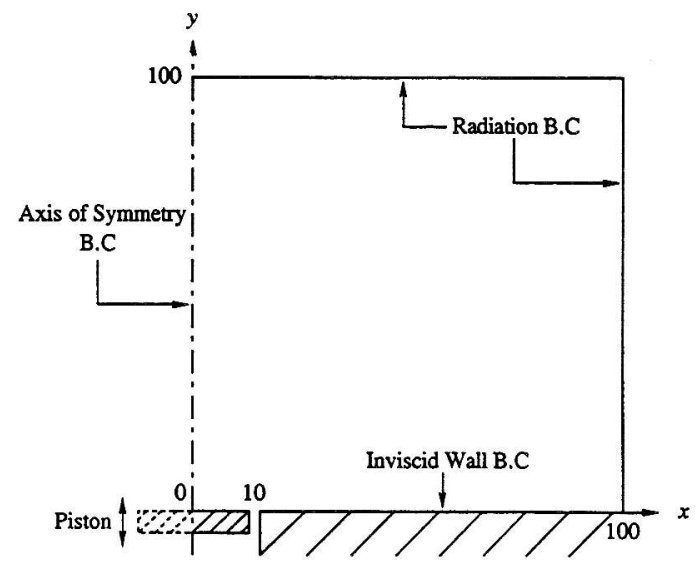

Fig. 3. Schematic diagram for the linear acoustic wave radiation problem: piston velocity $v=10^{-4} \sin (\pi t / 5)$.
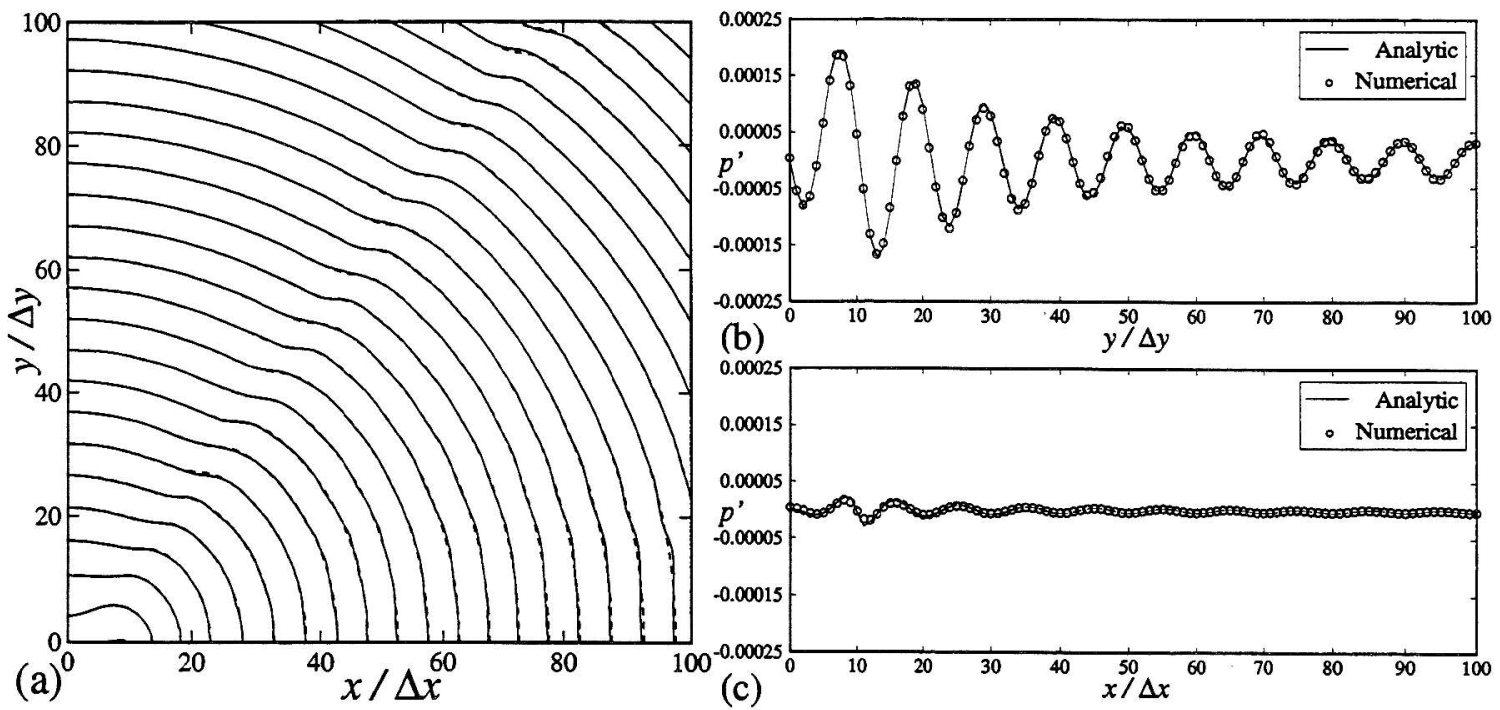

Fig. 4. Solutions of axisymmetric linear acoustic wave propagation and radiation by the OSOT scheme $(t / \Delta t=450)$ : (a) pressure contour ( - : numerical, - - - : analytic), (b) pressure fluctuation plot along $y$-axis $(x=0),(c)$ pressure fluctuation plot along $x$-axis $(y=0)$.

considerable reflecting waves, because the evaluation of density on the radiation boundaries is still based on the characteristics which have drawbacks in multi-dimensionality.

\subsection{Nonlinear waves}

An initial value problem is solved for the simulation of nonlinear acoustic wave propagation and radiation. The conservative forms of two-dimensional Euler equations are used for the computations. The numerical oscillations caused by the nonlinearity can be effectively removed by the addition of the adaptive selective damping (ASD) terms (see Refs. 4, 5 and 15 ) in the right hand side of Eq. (2.1). The results of computations by the OFOP scheme are 

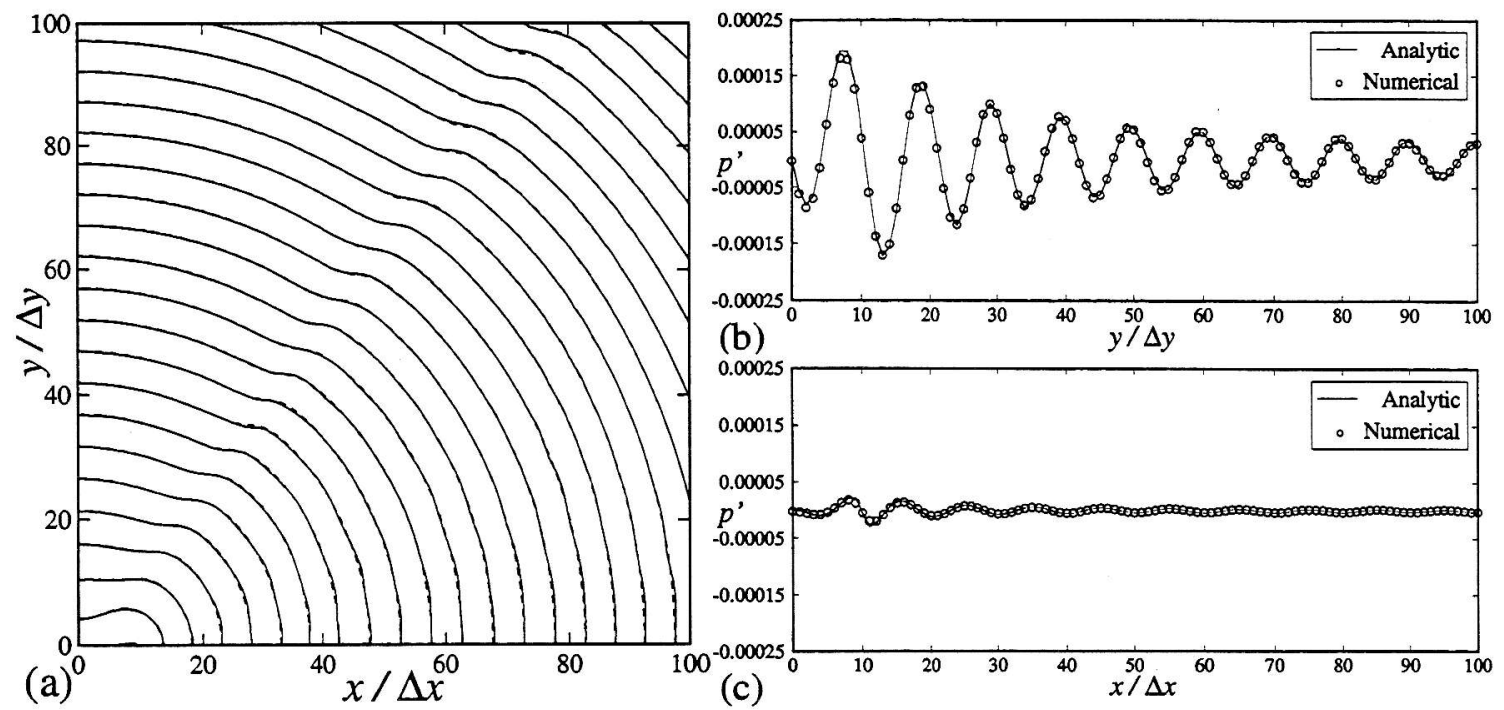

Fig. 5. Solutions of axisymmetric linear acoustic wave propagation and radiation by the OFOP scheme $(t / \Delta t=450)$ : (a) pressure contour (- : numerical, - - - : analytic), (b) pressure fluctuation plot along $y$-axis $(x=0)$, (c) pressure fluctuation plot along $x$-axis $(y=0)$.
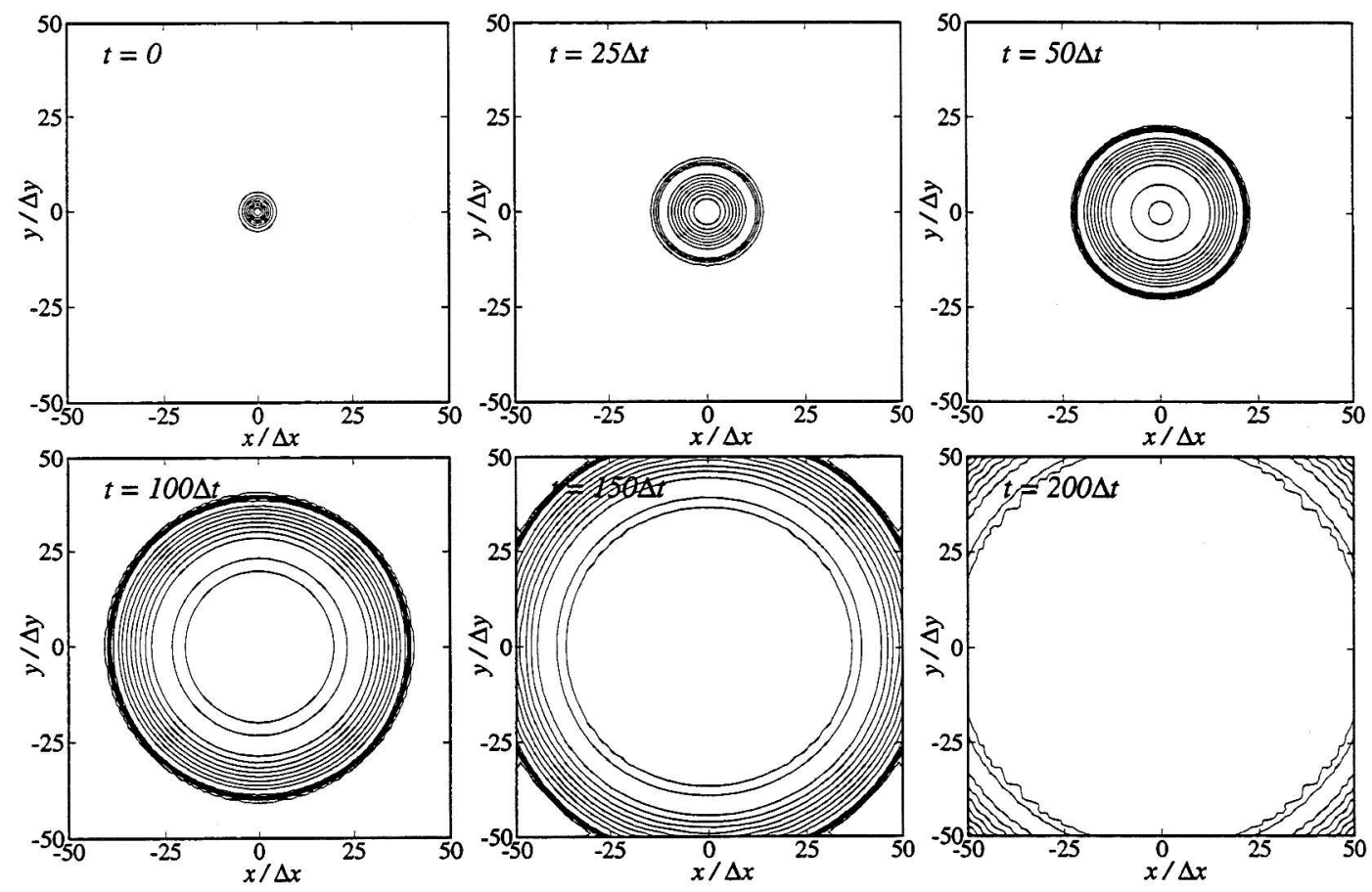

Fig. 6. Computation of nonlinear wave propagation and radiation by the OFOP scheme: pressure contours. 

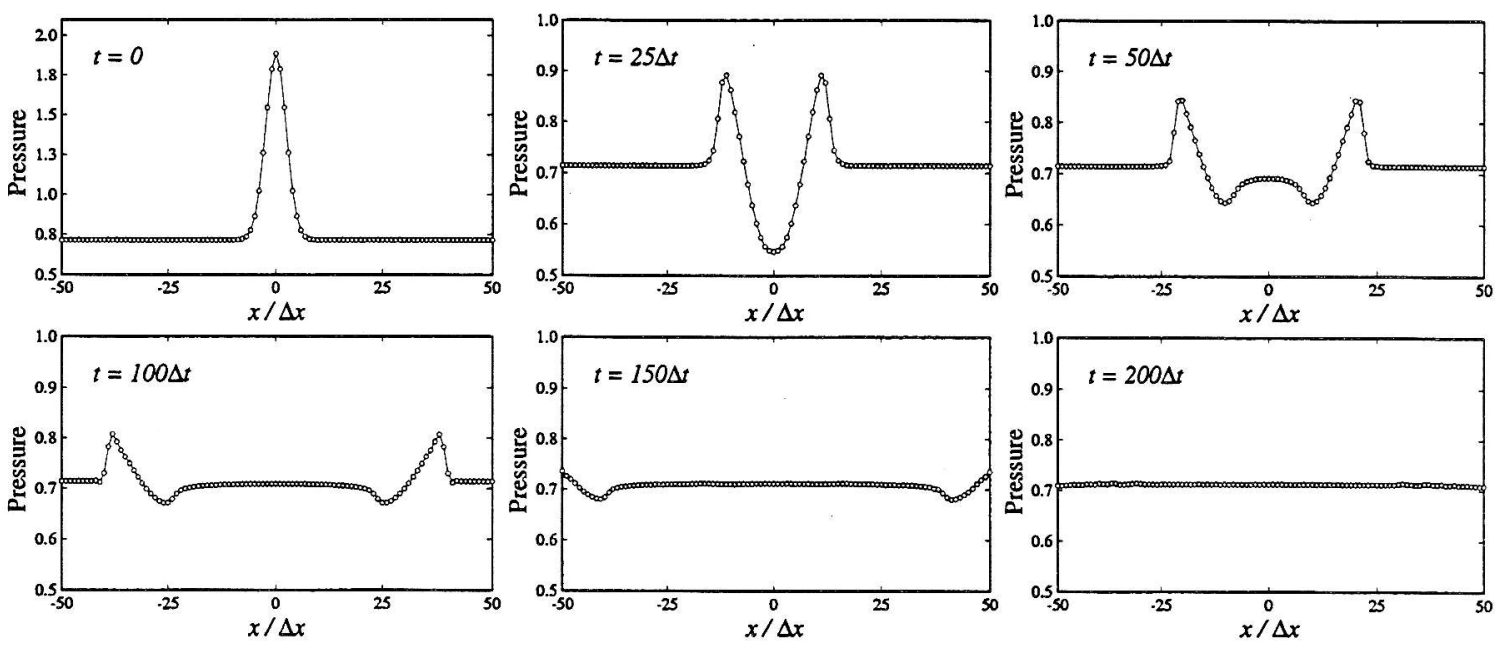

Fig. 7. Computation of nonlinear wave propagation and radiation by OFOP scheme: pressure plot along $x$-axis $(y=0)$.

presented in Figs. 6 and 7. With the non-reflecting radiation boundary conditions imposed. the computations are continued until the shock front passes through the far-field boundary, producing little reflecting waves. It is shown that the non-reflecting radiation boundary conditions considered here are used effectively for computations of the nonlinear wave as well as the linear wave.

\section{Conclusions}

The non-central near-boundary compact schemes are successfully optimized in the wavenumber domain to achieve maximum resolution characteristics. Analytic optimization methods are devised to minimize the dispersive and the dissipative errors of the nearboundary compact schemes. The optimized near-boundary compact schemes are effectively combined with the OHOC schemes for actual computations in a domain with non-periodic boundaries.

The characteristics-based boundary conditions and the free-field impedance conditions are proposed as the physical boundary conditions on an inviscid wall and non-reflecting radiation boundaries. These physical boundary conditions are used for the OH()C schemes to present high-quality numerical solutions. The linear and nonlinear wave propagation and radiation are accurately simulated by the OHOC schemes with the physical boundary conditions. In this way, the feasibility of implementing the physical boundary conditions for the $\mathrm{OHOC}$ schemes is investigated in this paper.

\section{References}

1. J. W. Kim and D. J. Lee, "Optimized compact finite difference schemes with maximum resolution," AIAA J. 34 (1996) to be published.

2. C. K. W. Tam and J. C. Webb, "Dispersion-relation-preserving schemes for computational acoustics," J. Computational Physics 107 (1993), 262-281. 
3. J. W. Kim and D. J. Lee, "Optimized compact finite difference schemes for solving wave equations," Proc. 1st CEAS/AIAA Aeroacoustics Conference, München, Germany, June 1995, 65-74.

4. J. W. Kim and D. J. Lee, "Optimized high-order compact schemes for linear and nonlinear wave generation and propagation," Proc. 4th International Congress on Sound and Vibration, St. Petersburg, Russia, June 1996.

5. J. W. Kim and D. J. Lee, "Numerical simulation of nonlinear waves using optimized high-order compact schemes," CFD J. accepted for publication.

6. S. K. Lele, "Compact finite difference schemes with spectral-like resolution," J. Computational Physics 107 (1993), 16-42.

7. R. Hixon, S. H. Shih and R. R. Mankbadi, "Evaluation of boundary conditions for computational aeroacoustics," 33rd AIAA Aerospace Science Meeting, Reno, NV, January 1995, AIAA-95-0160.

8. K. W. Thompson, "Time-dependent boundary conditions for hyperbolic systems," J. Computational Physics 68 (1987), 1-24.

9. K. W. Thompson, "Time-dependent boundary conditions for hyperbolic systems, II," J. Computational Physics 89 (1990), 439-461.

10. T. J. Poinsot and S. K. Lele, "Boundary conditions for direct simulations of compressible viscous flow," J. Computational Physics 101 (1992), 104-129.

11. M. B. Giles, "Non-reflecting boundary conditions for Euler equation calculations," AIAA J. 28 (1990), 2050-2058.

12. A. Bayliss and E. Turkel, "Far field boundary conditions for compressible flows," J. Computational Physics 48 (1982), 182-199.

13. T. Hagstrom and S. I. Hariharan, "Far field expansion for anisotropic wave equations," J. Computational Acoustics 2 (1990), 283-294.

14. D. J. Lee and S. O. Koo, "Numerical study of sound generation due to a spinning vortex pair," AIAA J. 33 (1995), 20-26.

15. C. K. W. Tam, J. C. Webb and Z. Dong, "A study of short wave components in computational acoustics," J. Computational Acoustics 1 (1993), 1-30.

16. F. Q. Fu, M. Y. Hussaini and J. Manthey, "Application of low dissipation and dispersion RungeKutta schemes to benchmark problems in computational aeroacoustics," Proc. ICASE/LaRC Workshop on Benchmark Problems in Computational Aeroacoustics, Hampton, Virginia, October 1994, 73-98.

17. Brower et al., Wave Motion 18 (1993), 383-400.

18. W. R. Watson and M. K. Myers, "Inflow-outflow boundary conditions for two-dimensional acoustic waves in channels with flow" AIAA J. 31 (1993), 1574-1582. 